\title{
Numerical Solution of Unsteady Hydromagnetic Couette Flow in a Rotating System Bounded by Porous Plates with Hall Effects
}

\author{
Muhim Chutia \\ Department of Mathematics \\ Mariani College, \\ Assam-785634, India
}

\author{
Tabendra Nath Das \\ Department of Mathematics \\ Dhakuakhana College \\ Assam-787055, India
}

\author{
Pranob Jyoti Chetia \\ Department of Mathematics \\ Duliajan College \\ Assam-786602
}

\begin{abstract}
Unsteady hydromagnetic Couette flow of a viscous, incompressible and electrically conducting fluid in a rotating system between two infinitely long parallel porous plates, taking Hall current into account, in the presence of a transverse magnetic field is studied numerically. Fluid flow within the channel is induced due to impulsive movement of the lower plate of the channel and fluid motion is subjected to a uniform suction and injection at upper and lower plates. Magnetic lines of force are assumed to be fixed relative to the fluid. Numerical solutions for primary and secondary velocities are obtained from the governing momentum equation by employing explicit finite difference method. The effects of various non-dimensional parameters: Hall current parameter $m$, magnetic parameter $M$, suction/injection parameter $S$ and time $t$ on primary and secondary velocities are presented graphically and discussed.
\end{abstract}

\section{Keywords}

MHD Couette flow, uniform suction/injection, Hall effects, rotating system, finite difference method.

\section{INTRODUCTION}

The study of unsteady magnetohydrodynamic (MHD) Couette flow in the presence of a transverse magnetic field has various and wide applications in many areas of science and engineering such as MHD pumps, MHD generators, MHD accelerators, MHD flow meters, nuclear reactors using liquid metal coolant etc. Katagiri [1] studied unsteady MHD Couette flow of a viscous, incompressible and electrically conducting fluid in the presence of a uniform transverse magnetic field when the fluid flow within the channel is induced due to impulsive movement of one of the plates of the channel. In recent years, the study of Couette flow in a rotating system enhances an interest to the researchers due to its applications in secular variation of earth's magnetic field, the internal rotation rate of sun, the structure of rotating magnetic stars, the planetary and solar dynamo problems, rotating hydromagnetic generators, vortex type MHD power generators and other centrifugal machines. Taking into account these facts, unsteady hydromagnetic Couette flow of a viscous incompressible electrically conducting fluid in a rotating system is investigated by Seth et al. [2,3,4,5], Chandran et al.[6], Hayat et al. [7], Das et al. [8], Jana et al. [9], Guria et al. [10] and Das et al. [11], under various aspects of the problem.

In all these investigations, the effects of Hall current are not taken into account. Cowling [12] stated that the effects of Hall current become significant if the strength of the magnetic field is high and number of density of electrons is small as it is responsible for the change of the flow pattern of an ionized gas. Hall Effect results in a development of an additional potential difference between opposite surfaces of a conductor for which a current is induced perpendicular to both the electric and magnetic field. Hall current induces secondary flow in the flow-field. Hall current on the fluid flow have many applications in MHD power generation, nuclear power reactors, underground energy storage systems, and in several areas of astrophysical and geophysical interest. Keeping in view of this fact, Jana and Datta [13], Mandal et al. [14], Ghosh and Pop [15], Hayat et al. [16], Guchhait et al [17], Ghosh [18], Reddy and Bathaiah [19], Das et al.[20], Chauhan and Agrawal [21], Seth et al. [22,23] and Harisingh Naik et al. [24] studied the effects of Hall current on MHD Couette flow of a viscous incompressible electrically conducting fluid in a rotating system considering different aspects of the problem. Hossain [25] studied unsteady hydromagnetic free convection flow near an infinite vertical porous plate with the effect of Hall current.

The study of MHD flow problem in porous channel may find applications in designing of cooling systems with liquid metals, geothermal reservoirs, underground energy transport, petroleum and mineral industries, in purification of crude oils etc. Taking into account this fact, Muhuri [26], Seth et al. [4,5,23], Guchhait [17], Reddy and Bathaiah [19], Harisingh Naik et al. [24] and Hossain [25] studied MHD Couette flow of electrically conducting fluid bounded by porous plates under different conditions, in rotating/ non-rotating system.

The purpose of the present paper is to investigate unsteady MHD Couette flow of a viscous, incompressible and electrically conducting fluid in a rotating system bounded by two parallel porous plates in the presence of transverse magnetic field with Hall effects. Fluid flow within the channel is induced due to impulsive movement of the lower plate of the channel. Numerical solution of the governing equation is obtained by finite difference method. The effects of rotation, Hall current, magnetic field, porosity of the plates and time on the flow-field have been analyzed and presented graphically.

\section{MATHEMATICAL FORMULATION}

Consider the unsteady MHD Couette flow of an electrically conducting, viscous, incompressible fluid between two parallel infinite horizontal porous plates $y^{\prime}=0$ and $y^{\prime}=a$ of infinite length in $x^{\prime}$ and $z^{\prime}$ directions. A uniform magnetic field $B_{0}$ is applied parallel to $y^{\prime}$-axis. The fluid as well as plates of the channel are in a state of rigid body rotation about $y^{\prime}$-axis with uniform angular velocity $\Omega^{\prime}$. Initially, at $t^{\prime}=$ $0\left(t^{\prime} \leq 0\right)$ both the fluid and plates are assumed to be at rest. But, at $t^{\prime}>0$, the lower plate $\left(y^{\prime}=0\right)$ starts to move with a constant velocity $U_{o}$ while the upper plate $\left(y^{\prime}=a\right)$ is stationary. There is no pressure gradient along the $x^{\prime}$-axis i.e $\partial p / \partial x^{\prime}=0$, and a uniform suction from above and injection 
from below which are applied at $t^{\prime}=0$. It is assumed that no applied and polarization voltage exists. This corresponds to the case where no energy is being added or extracted the fluid by electrical means (Meyer, [27]) i.e. electrical field, $\vec{E}=0$. Further, the induced magnetic field can be neglected by assuming a very small magnetic Reynolds number. The Hall Effect is taken into consideration and consequently a $z^{\prime}$ component for the velocity is expected to arise. The uniform suction and injection implies that the $y^{\prime}$-component of the velocity is constant i.e, $v^{\prime}=V_{0}$. Since the plates are infinite in the $x^{\prime}$ and $z^{\prime}$-directions, all the physical quantities do not change in these directions. Thus, the fluid velocity and magnetic field vectors are given by,

$\vec{V}=\left\{u^{\prime}\left(y^{\prime}, t^{\prime}\right), V_{0}, w^{\prime}\left(y^{\prime}, t^{\prime}\right)\right\}$ and

$\vec{B}=\left\{0, B_{0}, 0\right\}$

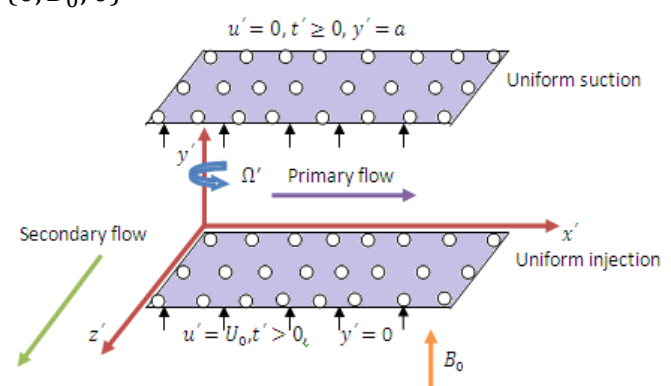

Fig 1: Geometrical configuration of the problem

The flow of the fluid is governed by the equations as follows:

Continuity equation:

$\nabla \cdot \vec{V}=0$

Momentum equation in a rotating system:

$\frac{\partial \vec{V}}{\partial t}+(\vec{V} \cdot \nabla) \vec{V}+2 \vec{\Omega} \times \vec{V}=-\frac{1}{\rho} \nabla p+v \nabla^{2} \vec{V}+\frac{1}{\rho}(\vec{J} \times \vec{B})$

Generalized Ohm's Law:

$\vec{J}=\sigma\left[\vec{E}+\vec{V} \times \vec{B}+\frac{1}{e n_{e}} \nabla P_{e}\right]-\frac{\omega \tau}{B_{0}}(\vec{J} \times \vec{B})$

Maxwell's Equations:

$\nabla \times \vec{E}=-\frac{\partial \vec{B}}{\partial t}$

$\nabla \cdot \vec{B}=0$

Here, $\vec{V}$ is the velocity field, $\vec{\Omega}$ is the angular velocity, $\vec{B}$ is the magnetic induction vector, $\vec{E}$ is the electric field vector, $\vec{J}$ is the current density vector, $p$ is the pressure of the fluid, $\rho$ is the density of the fluid, $\mu$ is the coefficient of viscosity, $v$ is the kinematic coefficient of viscosity, $e$ is the electron charge, $n_{e}$ is the number density of electron, $\omega$ is the electron cyclotron frequency, $\tau$ is the electron collision time, $P_{e}$ is the electron pressure and $t$ is the time.

We assume $\vec{E}$ to be negligible and the induced magnetic field can be ignored by assuming a very small magnetic Reynolds number. Moreover, in the absence of the ion-slip effects and electron pressure gradient; Generalized Ohm's Law [3] reduces to

$\vec{J}=\sigma(\vec{V} \times \vec{B})-\frac{m}{B_{0}}(\vec{J} \times \vec{B})$

where, $m=\omega \tau$ is the Hall parameter.

Using velocity $(\vec{V})$ and magnetic field $(\vec{B})$ as stated above, Eq. (6) can be solved in $\vec{J}$ to yield

$\vec{J} \times \vec{B}=-\frac{\sigma B_{0}^{2}}{1+m^{2}}\left[\left(u^{\prime}+m w^{\prime}\right) \hat{\boldsymbol{\imath}}+\left(w^{\prime}-m u^{\prime}\right) \widehat{\boldsymbol{k}}\right]$
Using (7), the $x^{\prime}$ and $z^{\prime}$-components of Eq. (2) can be written as

$$
\begin{aligned}
& \frac{\partial u^{\prime}}{\partial t^{\prime}}+V_{0} \frac{\partial u^{\prime}}{\partial y^{\prime}}+2 \Omega^{\prime} w^{\prime}=v \frac{\partial^{2} u^{\prime}}{\partial y^{\prime 2}}-\frac{\sigma B_{0}^{2}}{1+m^{2}}\left(u^{\prime}+m w^{\prime}\right) \\
& \frac{\partial w^{\prime}}{\partial t^{\prime}}+V_{0} \frac{\partial w^{\prime}}{\partial y^{\prime}}-2 \Omega^{\prime} u^{\prime}=v \frac{\partial^{2} w^{\prime}}{\partial y^{\prime 2}}-\frac{\sigma B_{0}^{2}}{1+m^{2}}\left(w^{\prime}-m u^{\prime}\right)
\end{aligned}
$$

The initial and boundary conditions for the problem are

$u^{\prime}=0, w^{\prime}=0$, at $t^{\prime} \leq 0$ for $0 \leq y^{\prime} \leq a$

$u^{\prime}=U_{0}, w^{\prime}=0$, at $y^{\prime}=0$ for all $\left.t^{\prime}>0\right\}$

$u^{\prime}=0, w^{\prime}=0$, at $y^{\prime}=a$ for all $\left.t^{\prime}>0\right\}$

We, introduce the following non-dimensional quantities

$y=\frac{y^{\prime}}{a}, u=\frac{u^{\prime}}{U_{0}}, w=\frac{w^{\prime}}{U_{0}}, t=\frac{v t^{\prime}}{a^{2}}$

Using non-dimensional quantities (11) in Eqs. (8) and (9), we obtain

$\frac{\partial u}{\partial t}+S \frac{\partial u}{\partial y}+2 \Omega w=\frac{\partial^{2} u}{\partial y^{2}}-\frac{M^{2}}{1+m^{2}}(u+m w)$

$\frac{\partial w}{\partial t}+S \frac{\partial w}{\partial y}-2 \Omega u=\frac{\partial^{2} w}{\partial y^{2}}-\frac{M^{2}}{1+m^{2}}(w-m u)$

where, $S=\frac{V_{o} a}{v}$ is the suction or injection parameter $(S>0$ for suction and $S<0$ for injection), $M=B_{o} a\left(\frac{\sigma}{\rho v}\right)^{1 / 2}$ is the Hartmann number and $\Omega=\frac{\Omega^{\prime} a^{2}}{v}$ is the rotation parameter.

The initial and boundary conditions (10), in dimensionless form, become

$\left.\begin{array}{l}u=0, w=0, \text { at } t \leq 0 \text { for } 0 \leq \mathrm{y} \leq 1 \\ u=1, w=0, \text { at } y=0 \text { for all } t>0 \\ u=0, w=0, \text { at } y=1 \text { for all } \mathrm{t}>0\end{array}\right\}$

\section{NUMERICAL SOLUTION}

Equations (12) and (13) represent a system of coupled linear partial differential equations which can be solved numerically subject to the initial and boundary conditions (14) using finite difference approximations. In numerical procedure computational domain is divided into a uniform grid system. Both the second-derivative and the first-derivative terms for space and time are discretized using the central difference approximations of second-order accuracy. The finite difference equations corresponding to the governing Eqs. (12) and (13) are as follows

$$
\begin{aligned}
& \frac{u_{i, j+1}-u_{i, j-1}}{2 k}+S\left(\frac{u_{i+1, j}-u_{i-1, j}}{2 h}\right)+2 \Omega w_{i, j} \\
& =\frac{u_{i+1, j}-2 u_{i, j}+u_{i-1, j}}{h^{2}}-\frac{M^{2}}{1+m^{2}}\left(u_{i, j}+m w_{i, j}\right) \\
& \frac{w_{i, j+1}-w_{i, j-1}}{2 k}+S\left(\frac{w_{i+1, j}-w_{i-1, j}}{2 h}\right)-2 \Omega u_{i, j} \\
& =\frac{w_{i+1, j}-2 w_{i, j}+w_{i-1, j}}{h^{2}}-\frac{M^{2}}{1+m^{2}}\left(w_{i, j}-m u_{i, j}\right)
\end{aligned}
$$

Then the initial and boundary conditions (14) become

$$
\left.\begin{array}{l}
u_{i, 0}=0, \quad w_{i, 0}=0, \text { at } j \leq 0 \text { for } i \neq 0 \\
u_{0, j}=1, w_{0, j}=0, \text { at } i=0 \text { for all } j>0 \\
u_{N, j}=0, w_{N, j}=0 \text {, at } i=N \text { for all } j>0
\end{array}\right\}
$$

where, index $i$ refers to $y, j$ refers to $t$ and $N$ denotes number of grids inside the computational domain in the $Y$-direction.

Solving Eqs. (15) and (16) respectively for $u_{i, j}$ and $w_{i, j}$, we obtain the following equations 


$$
\begin{aligned}
u_{i, j}= & C_{1}\left(u_{i+1, j}+u_{i-1, j}\right)-C_{2}\left(u_{i, j+1}-u_{i, j-1}\right) \\
& -C_{3}\left(u_{i+1, j}-u_{i-1, j}\right)-C_{4} w_{i, j} \\
w_{i, j}= & C_{1}\left(w_{i+1, j}+w_{i-1, j}\right)-C_{2}\left(w_{i, j+1}-w_{i, j-1}\right) \\
& -C_{3}\left(w_{i+1, j}-w_{i-1, j}\right)+C_{4} u_{i, j}
\end{aligned}
$$

where,

$C_{1}=\frac{1}{2+\frac{h^{2} M^{2}}{1+m^{2}}}, C_{2}=\frac{h^{2}}{2 k\left(2+\frac{h^{2} M^{2}}{1+m^{2}}\right)}, C_{3}=\frac{h S}{2\left(2+\frac{h^{2} M^{2}}{1+m^{2}}\right)}$,

$C_{4}=\frac{h^{2}\left(2 \Omega+\frac{m M^{2}}{!+m^{2}}\right)}{2+\frac{h^{2} M^{2}}{1+m^{2}}}$ are constants.

The numerical solutions of Eqs. (18) and (19) subject to the initial and boundary conditions (17) are obtained by first selecting non-dimensional parameters that are involved such as $M, m, \Omega$ and $S$. Then knowing the values of $u$ and $w$ at a time $t$, we can evaluate the values at a time $t+\Delta t$ as follows. We substitute $i=1,2,3, \cdots \cdots \cdots, N-1$ in the Eq. (18) which constitutes a tri-diagonal system of linear equations; the system can be solved by Gauss Seidal iteration method (Mathews and Fink, [28]). Thus $u$ is known for all values of $y$ at time $t$. Then knowing values of $u$ and applying the same procedure with the boundary conditions (17), we calculate $w$ from equations (19). This procedure is continued to obtain the solution till the converged solutions for $u$ and $w$ in the grids system are obtained at desired time $t$.

\section{RESULTS AND DISCUSSION}

In this present paper, Hall Effect on unsteady MHD Couette flow of an electrically conducting viscous incompressible fluid in a rotating system bounded by two parallel nonconducting porous plates in the presence of transverse magnetic field have been investigated numerically. Numerical solutions for the non-dimensional fluid velocity components $u$ and $w$ have been presented for different values of the Hartmann number $(M)$, rotation parameter $(\Omega)$, Hall parameter $(m)$, suction/injection parameter $(S)$, and time $t$ against $y$ in Figs. 2-14.

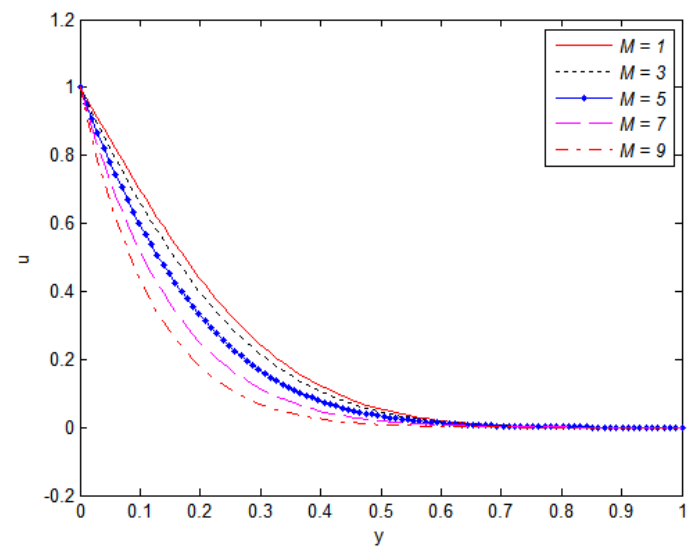

Fig 2: Primary velocity profile $u$ at various $M$ when $S=1$, $m=0.5, \Omega=2$ and $t=0.03$

In Fig. 2 and Fig. 3, we have presented the primary and secondary velocity profiles for different values of the Hartmann number $M$ keeping other parameters fixed. It is seen in these figures that the magnitude of the primary velocity $u$ decreases and the secondary velocity $w$ increases with an increase in Hartmann number $M$. It is clear from Eq. (12) that the term $\frac{m M^{2}}{1+m^{2}} u$, which decides the flow in the $z$ - direction. If the Hartmann number $M=0$, then the term mentioned above is zero and hence there is no force to induce the flow in the $z$-direction, that is, $w=0$. The interaction of the transverse magnetic field with the moving fluid creates a body force called Lorentz force, and plays the role of a resistive type force on the primary flow similar to a drag force that acts in the opposite direction of the fluid motion and tends to retard the flow thereby reducing the primary velocity. On the other hand, the resulting Lorentz force will not act as a drag force but act as an aiding body force on the secondary flow. This will serve to accelerate the secondary velocity. This is in good agreement with the results of Hossain [25].

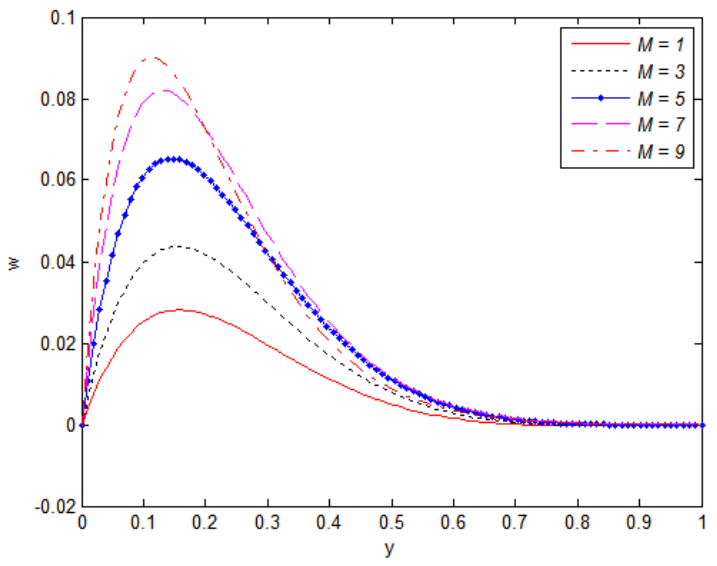

Fig 3: Secondary velocity profile $w$ at various $M$ when $S=1, m=0.5, \Omega=2$ and $t=0.03$

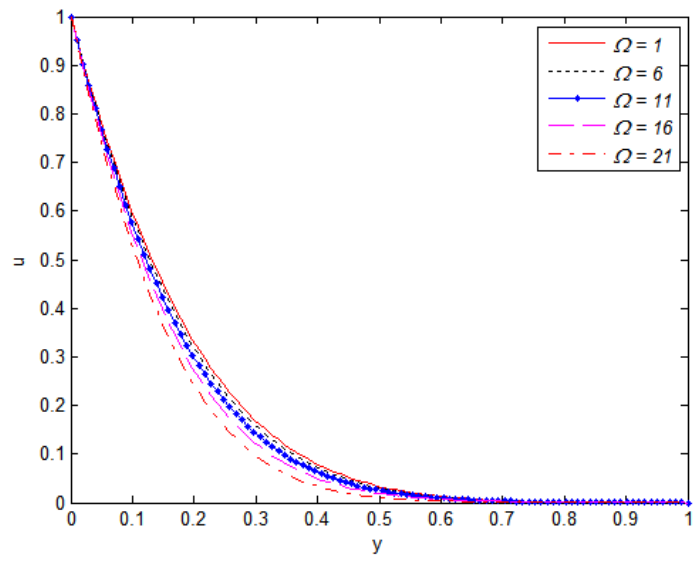

Fig 4: Primary velocity profile $u$ at various $\Omega$ when $S=1$, $M=5, m=0.5$ and $t=0.03$

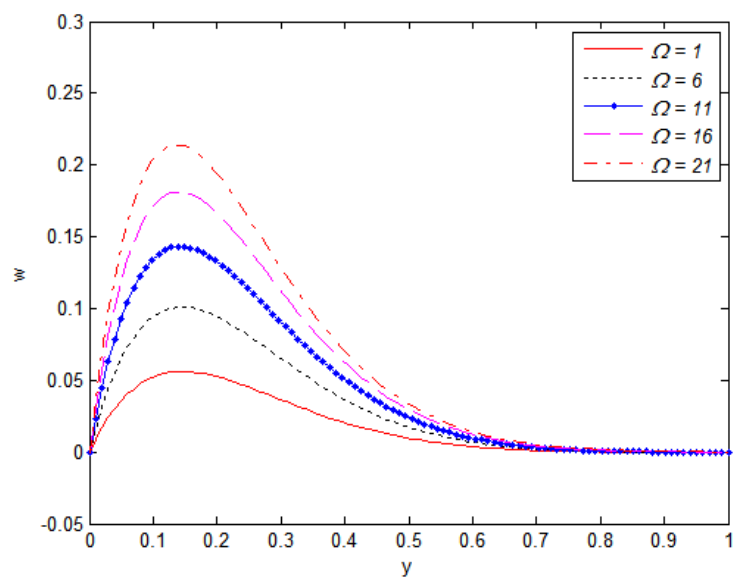

Fig 5: Secondary velocity profile $w$ at various $\Omega$ when $S=1, M=5, m=0.5$ and $t=0.03$ 


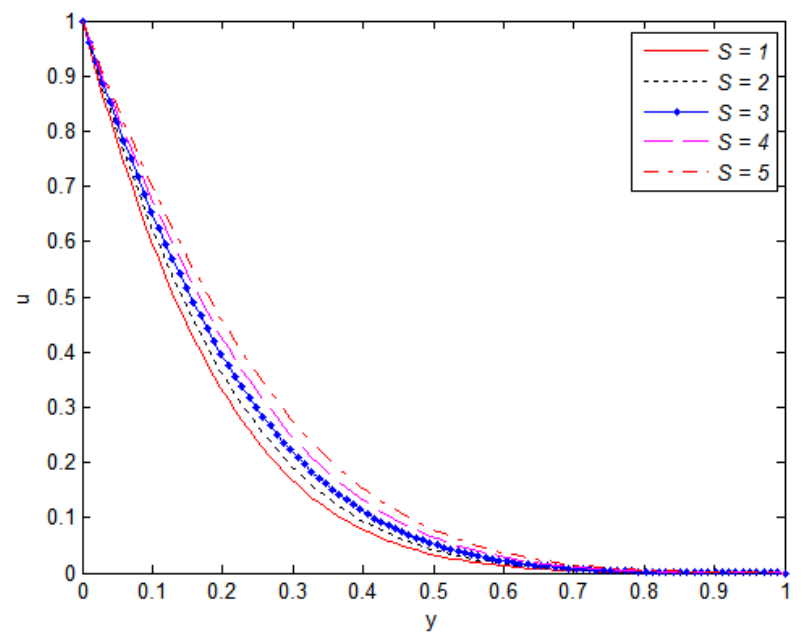

Fig 6: Primary velocity profile $u$ at various $S(S>0)$ when $M=5, m=0.5, \Omega=2$ and $t=0.03$

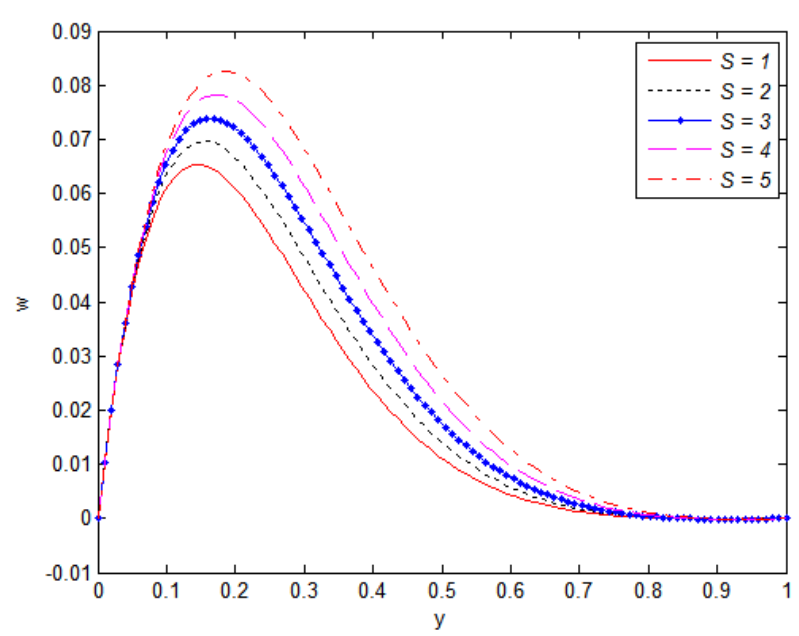

Fig 7: Secondary velocity profile $w$ at various $S(S>0)$ when $M=5, m=0.5, \Omega=2$ and $t=0.03$

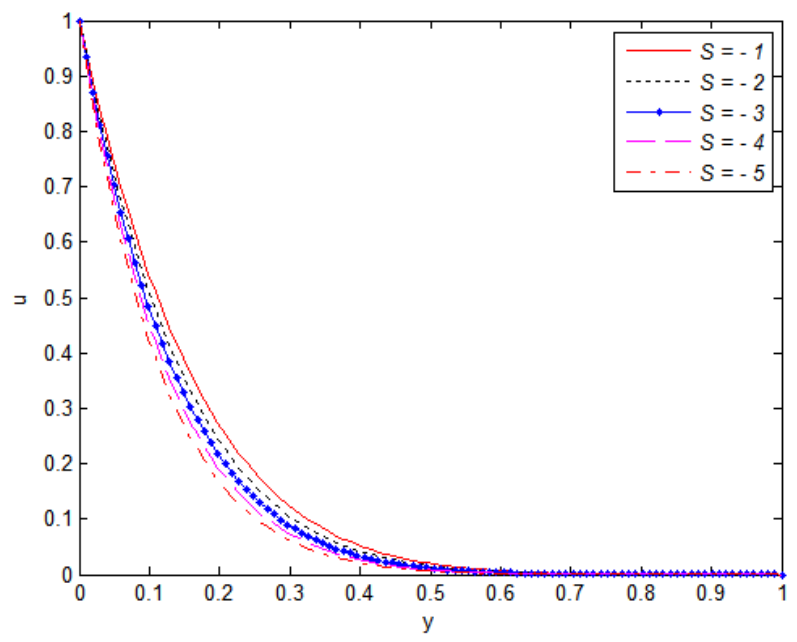

Fig 8: Primary velocity profile $\boldsymbol{u}$ at various $\boldsymbol{S}(\boldsymbol{S}<0)$ when $M=5, m=0.5, \Omega=2$ and $t=0.03$

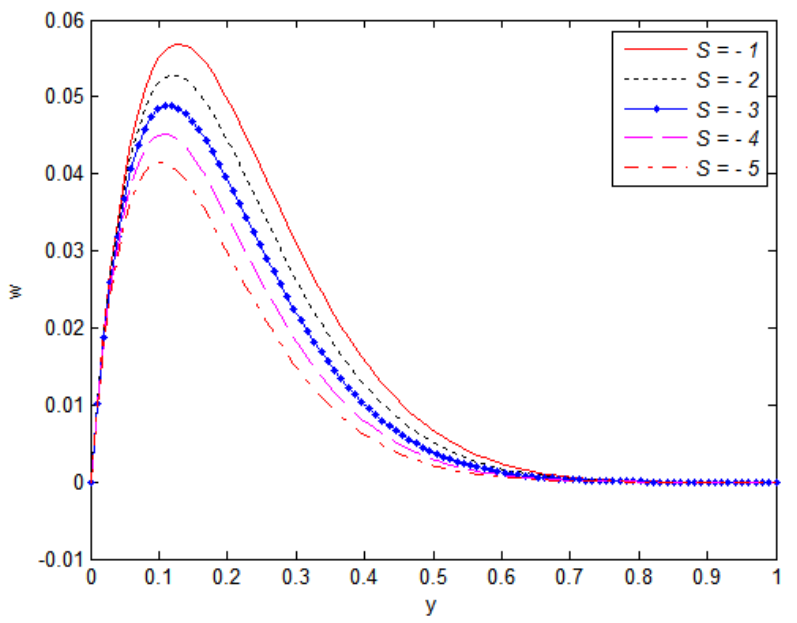

Fig 9: Secondary velocity profile $\boldsymbol{w}$ at various $S(S<0)$ when $M=5, m=0.5, \Omega=2$ and $t=0.03$

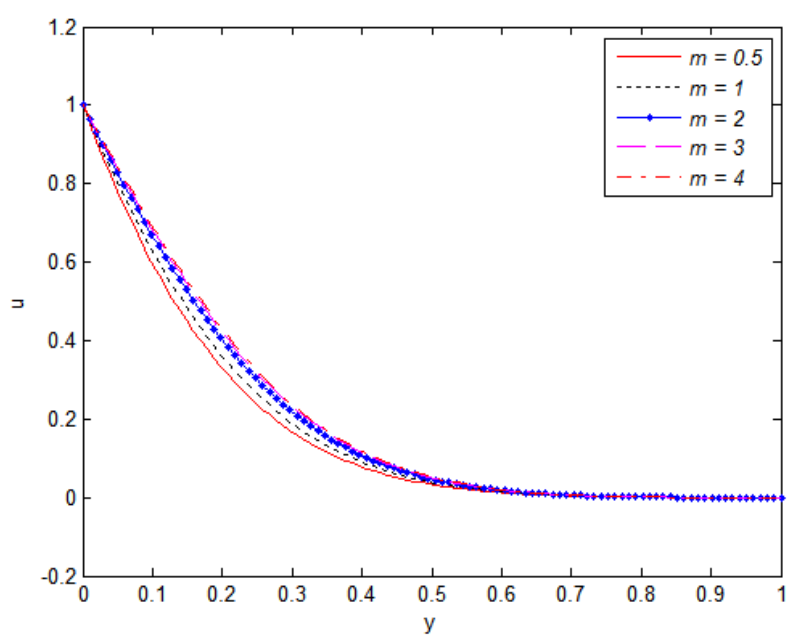

Fig 10: Primary velocity profile $u$ at various $m$ when $S=1, M=5, \Omega=2$ and $t=0.03$

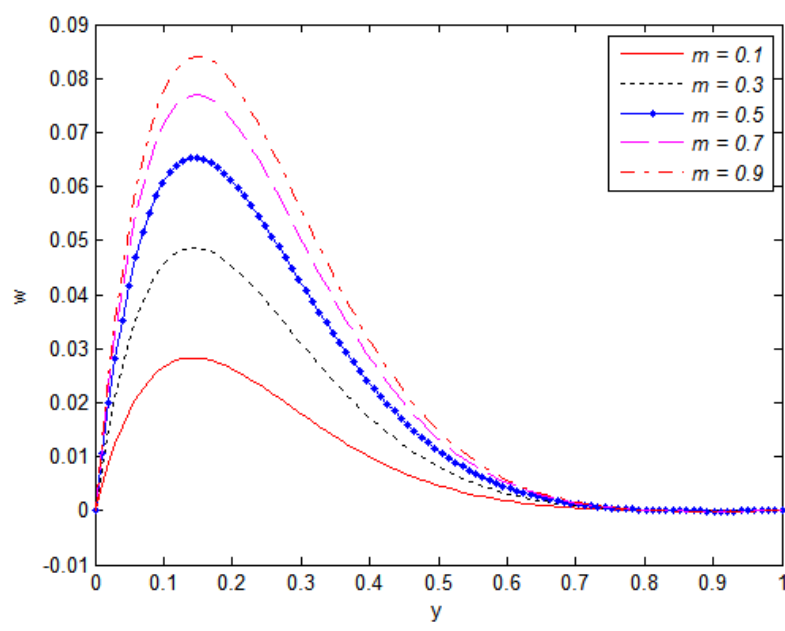

Fig 11: Secondary velocity profile $\boldsymbol{w}$ at various $\boldsymbol{m}(\boldsymbol{m}<1)$ when $S=1, M=5, \Omega=2$ and $t=0.03$ 


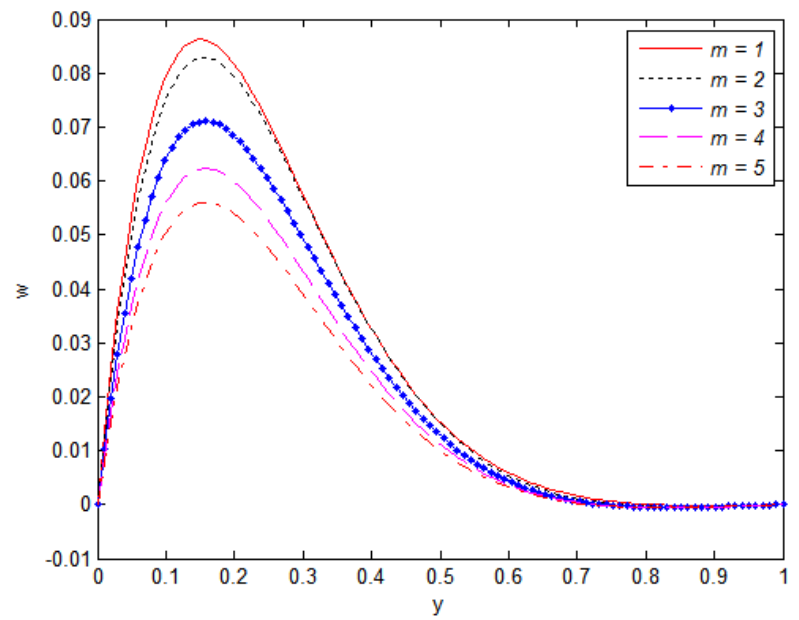

Fig 12: Secondary velocity profile $w$ at various $m(m \geq 1)$ when $S=1, M=5, \Omega=2$ and $t=0.03$

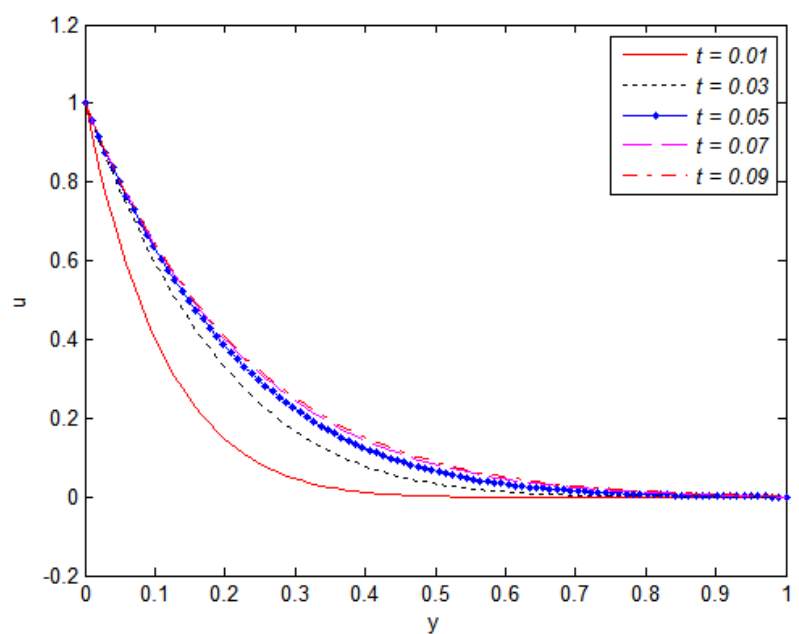

Fig 13: Primary velocity profile $u$ at various $t$ when $S=1$, $M=\mathbf{5}, \boldsymbol{m}=\mathbf{0 . 5}$ and $\Omega=\mathbf{2}$

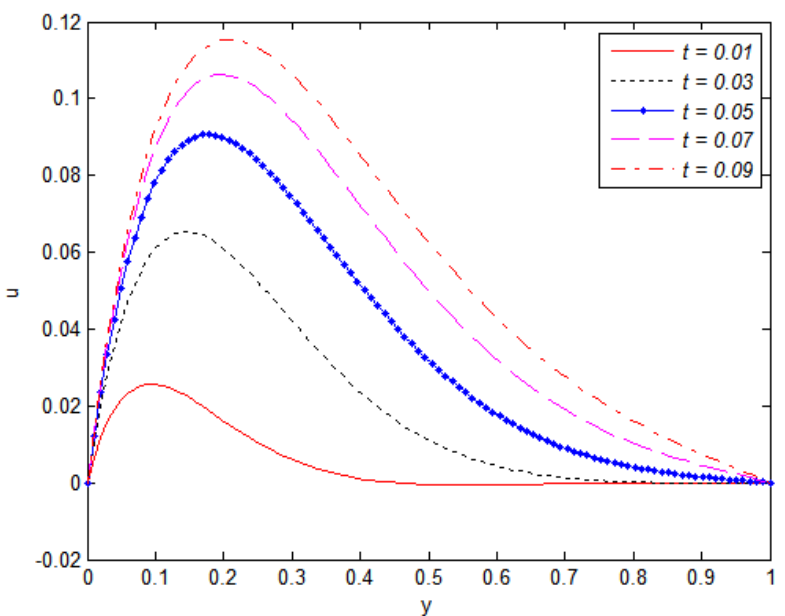

Fig 14: Secondary velocity profile $w$ at various $t$ when $S=1, M=5, m=0.5$ and $\Omega=2$

Fig. 4 and Fig. 5 present the effects of rotation parameter $\Omega$ on primary and secondary velocities for fixed values of $S=1$, $M=5, m=0.5$ and $t=0.03$ respectively. It is observed in Fig. 4 that the primary velocity $u$ decreases with an increase in rotation parameter $\Omega$. While, the secondary velocity $w$ increases for increasing values $\Omega$ as it is seen in Fig. 5. It confirms the result obtained by Seth et al. [5].
The effects of suction parameter $S(S>0)$ on velocity components $u$ and $w$ are depicted in Fig. 6 and Fig. 7 respectively, when $M=5, m=0.5, \Omega=2$ and $t=0.03$. It is observed in Fig. 6 and Fig. 7, that both primary and secondary velocity increases as suction parameter $S$ increases.

Similarly, the effects of injection parameter $S(S<0)$ on velocity components $u$ and $w$ are depicted in Fig. 8 and Fig. 9 respectively. The variation of velocity profiles for the injection case is quite opposite to that of suction case as shown in Fig. 6- Fig. 7.

Fig. 10 demonstrates the primary velocity $u$ for different values of the Hall parameter $m$, when $S=1, M=5, \Omega=2$ and $t=0.03$. It is observed that the Hall current promotes the flow along the channel. So, primary velocity $u$ increases with an increase in Hall parameter. This is because, in general, the Hall currents reduce the resistance offered by the Lorentz force.

The effect of Hall current on the secondary velocity $w$ is depicted in Fig. 11 and Fig. 12 for the cases $m<1$ and $m \geq 1$ respectively. The secondary velocity is induced by the component of the Lorentz force in the $z$-direction which arises solely due to the Hall current. From Eq. (12), it is clear that the term $\frac{m}{1+m^{2}} u$ decides the flow in the $z$-direction. If the Hall parameter $m=0$, then the term mentioned above is zero and hence there is no force to induce the flow in the $z$-direction. That is $w=0$. Further, $\frac{m}{1+m^{2}}$ increases as $m$ increases in the range $0 \leq m \leq 1$, and it decreases as $m$ increases in the range $m>1$. This implies that the magnitude of the component of the Lorentz force in the $z$-direction increases as $m$ increases in the range $0 \leq m \leq 1$ and hence the secondary velocity $w$ increases, while it decreases when $m$ increases in the range $m>1$ and hence the secondary velocity $w$ is decreases. These results are analyzed graphically in Fig. 11 and Fig. 12. These are in good agreement with the result obtained by Harisingh Naik et al. [24].

Fig. 13 and Fig. 14 present the velocity components $u$ and $w$ as functions of $y$ for different values of the time starting from $t=0$ to the steady state when $S=1, M=5$ and $m=1$. It is observed in Fig. 13 and Fig. 14 that both the primary and secondary velocity components $u$ and $w$ increase with an increase in time and reach the steady state monotonically and $u$ reaches the steady state faster than $w$.

\section{CONCLUSIONS}

In this paper, unsteady MHD Couette flow of a viscous, incompressible and electrically conducting fluid in a rotating system bounded by two infinitely long parallel porous plates, taking Hall current into account, in the presence of a transverse magnetic field is studied numerically by explicit finite difference method. Based on the computed results presented above in terms of graphics the following conclusions are made.

a. Effect of applied magnetic field is to retard the primary flow throughout the channel and to support the secondary flow induced by the Hall current.

b. The effect of rotation is to reduce the primary flow and enhance the secondary flow throughout the channel.

c. Hall current promotes primary flow throughout the channel; it enhances the secondary flow when the Hall parameter is increased up to unity and retards the secondary flow when Hall parameter is increased beyond unity. 
d. The suction parameter $S(S>0)$ enhances both primary flow and secondary flow, while the injection parameter $S(S<0)$ has a reversal effects on flow variables.

e. Both the primary and secondary flows increase with an increase in time and reach the steady state monotonically and primary flow reaches the steady state faster than secondary flow.

\section{REFERENCES}

[1] Katagiri, M. 1962. Flow formation in Couette motion in magnetohydrodynamics. Journal of Physical Society of Japan 17, 393-396.

[2] Seth, G. S., Jana, R. N. and Maiti, M. K. 1982. Unsteady hydromagnetic Couette flow in a rotating system. Int. J. Engng. Sci. 20, 989-999.

[3] Seth, G. S., Nandkeolyar, R., Mahto, N. and Singh, S. K. 2009. MHD Couette flow in a rotating system in the presence of inclined magnetic field. Appl Math. Sci. 3, 2919-2932.

[4] Seth, G. S., Ansari, Md. S and Nandkeolyar, R. 2010. Unsteady hydromagnetic Couette flow within porous plates in a rotating system. Adv. Appl. Math. Mech. 2, 286-302.

[5] Seth, G. S., Ansari, Md. S. and Nandkeolyar, R. 2011. Effects of rotation and magnetic field on unsteady Couette flow in a porous channel. Journal of Applied Fluid Mechanics 4(2), 95-103.

[6] Chandran, P., Sacheti, N. C. and Singh, A. K. 1993. Effect of rotation on unsteady hydromagnetic Couette flow. Astrophys. Space Sci. 202, 1-10.

[7] Hayat, T., Nadeem, S. and Asghar, S. 2004. Hydromagnetic Couette flow of an Oldroyd-B fluid in a rotating system. Int. J. Engng. Sci. 42, 65-78.

[8] Das, S., Maji, S. L., Guria, M. and Jana, R. N. 2009. Unsteady MHD Couette flow in a rotating system. Math. Comp. Modelling 50, 1211-1217.

[9] Jana, M., Das, S. and Jana, R. N. 2012. Unsteady Couette Flow through a Porous Medium in a Rotating System. Open Journal of Fluid Dynamics 2, 149-158.

[10] Guria, M., Jana, R. N. and S.K. Ghosh, S. K. 2006. Unsteady Couette flow in a rotating system. International journal of Non-Linear Mechanics 41, 838-843.

[11] Das, B .K., Guria, M. and Jana, R. N. 2008. Unsteady Couette flow in a rotating system. Mechanics 43, 517521.

[12] Cowling, T. G. 1957. Magnetohydrodynamics. p. 101. Interscience, New York.

[13] Jana, R. N. and Datta, N. 1980. Hall effects on MHD Couette flow in a rotating system. Czech. J. Phys. B 30, 659-667.
[14] Mandal, G., Mandal , K. K. and Choudhury, G. 1982. On combined effects of Coriolis force and Hall current on steady MHD Couette flow and heat transfer. J. Phys. Soc. Japan 51, 2010-2015

[15] Ghosh, S. K. and Pop, I. 2004. Hall effects on MHD plasma Couette flow in a rotating environment. Int. J. Appl. Mech. Engng. 9, 293-305.

[16] Hayat, T., Wang, Y. and Hutter, K. 2004. Hall effects on the unsteady hydromagnetic oscillatory flow of a secondgrade fluid. Int. J. Non-linear Mech. 39, 1027-1037.

[17] Guchhait, S., Das, S., Jana, R. N. and Ghosh, S. K. 2011. Combined Effects of Hall Current and Rotation on Unsteady Couette Flow in a Porous Channel, World Journal of Mechanics 1, 87-99.

[18] Ghosh, S.K. 2002. Effects of Hall Current on MHD Couette Flow in a Rotating System with Arbitrary Magnetic Field. Czechoslovak Journal of Physics, 52(1). 51-63.

[19] Reddy, N. B. and Bathaiah, D. 1982. Hall Effects on MHD Couette Flow through a Porous Straight Channel. Defense Science Journal 32, 313-326.

[20] Das, S., Sarkar, B. C. and Jana, R. N. 2011. Hall Effects on MHD Couette Flow in Rotating System. International Journal of Computer Applications 3, 22-30.

[21] Chauhan, D. S. and Agrawal, R. 2012. Effects of Hall current on MHD Couette flow in a channel partially filled with a porous medium in a rotating system. Meccanica 47, 405-421.

[22] Seth, G.S., Nandkeolyar, R. and Ansari, Md. S. 2009. Hall effects on oscillatory hydromagnetic Couette flow in a rotating system. Int. J. Acad.Res. 1, 6-17.

[23] Seth, G. S., Singh, J. K. and Mahato, G. K. 2012. Effects of Hall current and rotation on unsteady hydromagnetic Couette flow within a porous channel. International Journal of Applied Mechanics 4, 1250015(25 pages).

[24] Harisingh Naik, S., Ramana Murthy, M. V. and Rama Rao, K. 2014. The effect of Hall current on an unsteady MHD free convective Couette flow between two permeable plates in the presence of thermal radiation. International Journal of Computational Engineering Research 4(7), 2250-3005.

[25] Hossain, M. A. 1986. Effect of Hall current on unsteady hydromagnetic free convection flow near an infinite vertical porous plate. Journal of the Physical Society of Japan 55, 2183- 2190.

[26] Muhuri, P. K. 1963. Flow formation in Couette motion in Magnetohydrodynamics with suction. J. Phys. Soc. Jpn. 18, 1671- 1675 .

[27] Meyer, R. C. 1958. On reducing aerodynamic heat transfer rates by magnetohydrodynamic techniques. J. Aero. Sci. 25, 561-572.

[28] Mathews, J. H. and Fink, K. D. 2009. Numerical Method using Matlab. PHI Learning Private Limited, New Delhi. 